\title{
Gauge and Supersymmetric Invariance of a Boundary Bagger-Lambert-Gustavsson Theory
}

\author{
Mir Faizal \\ Mathematical Institute, University of Oxford \\ Oxford OX1 3LB, United Kingdom
}

November 21, 2018

\begin{abstract}
In this paper we will discuss the effect of a having a boundary on the supersymmetric invariance and gauge invariance of the Bagger-LambertGustavsson (BLG) Theory. We will show that even though the supersymmetry and gauge invariance of the original BLG theory is broken due to the presence of a boundary, it restored by the addition of suitable boundary terms. In fact, to achieve the gauge invariance of this theory, we will have to introduce new boundary degrees of freedom. The boundary theory obeyed by these new boundary degrees of freedom will be shown to be a generalization of the gauged Wess-Zumino-Witten model, with the generators of the Lie algebra replaced by the generators of the Lie 3 -algebra. The gauge and supersymmetry variations of the boundary theory will exactly cancel the boundary terms generated by the gauge and supersymmetric variations of the bulk theory.
\end{abstract}

\section{Introduction}

Bagger-Lambert-Gustavsson (BLG) theory is a superconformal field theory with $\mathcal{N}=8$ supersymmetry $[1,2,3,4,5$. This is thought to be the theory that describes the low energy behavior of would volume of multiple $M 2$-branes. In this theory fields take value in a Lie 3 -algebra rather than a regular Lie algebra. The BLG theory has been analysed in $\mathcal{N}=1$ superspace [6, 7], $\mathcal{N}=2$ superspace [8, 9, and $\mathcal{N}=8$ superspace [10, 11. In this paper we will perform our analysis in $\mathcal{N}=1$ superspace. Higgs mechanism for the BLG theory has also been studied in $\mathcal{N}=1$ superspace formalism [7]. Thus, higher derivative corrections to super-Yang-Mills on $D 2$-branes have been analysed in $\mathcal{N}=1$ superspace.

Just like in string theory, strings can end on $D$-branes, in $M$-theory $M 2$ branes can end on $M 5$-branes. So, the $M 2$-brane can be viewed as string like soliton of the non-linear world volume equations of motion. In fact, this soliton is known as the self dual string [12. In analogy with $D 1-D 3$ system this the ending can also be described as a fuzzy $S^{3}$ funnel solution of the Basu-Harvey equation [13, 14, 15]. Thus, by studding $M 2$-branes with boundaries, we can understand the dynamics of $M 5$-branes. In fact, the equation of motion of a $M 5$ - 
brane have been derived by demanding the $\kappa$-symmetry of the open membrane action [16, 17.

In addition to this $M 2$-branes ending on $M 9$-branes and gravitational waves have also been analysed [18. Boundary effects for the BLG theory with fluxes, on a manifold with boundaries, have been studied 19, 20. The fact that the fields take values in a Lie 3-algebra is crucial in the construction of the the BLG theory. However, only one example of a Lie 3-algebra is known and it has been difficult to increase the rank of the gauge group. So, usually another theory called the ABJM theory is studied as the theory of multiple $M 2$-branes 21. This theory has only manifest $\mathcal{N}=6$ supersymmetry, but it is expected to be enhanced to full $\mathcal{N}=8$ supersymmetry 22 . This theory agrees with the BLG theory for the only example of the Lie 3-algebra known 23]. The supersymmetric and gauge invariance of the ABJM theory in presence of boundaries has been already been studied. In fact, a gauge invariant model has been constructed by coupling the bosonic ABJM in presence of boundaries to a gauged Wess-Zumino-Witten model living on the boundary 24. The supersymmetric invariance of general field theories in presence of boundaries has been studied in $\mathcal{N}=1$ superspace formalism [25]. This formalism has also been used to analyse the supersymmetry of the gauge part of the ABJM theory [26.

In this paper we will analyse the BLG theory with a boundary in $\mathcal{N}=$ 1 superspace formalism. We will observe that like the ABJM theory, both supersymmetric and gauge invariant is broken due to the presence of a boundary. However if we suitable coupled the bulk BLG theory to a boundary theory, the resultant theory can be made both gauge invariant and supersymmetric. We will find that the theory that is needed to make the bulk BLG theory gauge invariant is a generalization of the gauged Wess-Zumino-Witten model, with the gauge symmetries generated by the Lie 3-algebra.

\section{Super-Covariant Derivatives}

Three dimensional non-abelian supergauge theory in $\mathcal{N}=1$ superspace formalism has been throughly analysed 28 and boundary effects in abelian supergauge theory have also been studied 25]. In this section we will analyse the boundary effects in supergauge theory, with the gauge symmetry generated by generators of the Lie 3-algebra. To do so we first review some properties of a Lie 3-algebra 27. A Lie 3 -algebra $\mathcal{A}$ is defined by

$$
\left[T^{A}, T^{B}, T^{C}\right]=f_{D}^{A B C} T^{D} .
$$

where $T^{A}$ are the generators of this Lie 3-algebra and $f_{D}^{A B C}$ are the structure constants. These structure constants are totally antisymmetric in $A, B, C$ and are subject to the fundamental identity resembling the Jacobi identity for Lie algebras,

$$
f_{G}^{[A B C} f_{H}^{D] E G}=0 .
$$

It is useful to define another constant constructed from these structure constant as

$$
C_{E F}^{A B, C D}=2 f_{[E}^{A B[C} \delta_{F]}^{D]} .
$$


These constants are anti-symmetric in the pair of indices $A B$ and $C D$ and also satisfy a kind of Jacobi identity [27,

$$
C_{E F}^{A B, C D} C_{K L}^{G H, E F}+C_{E F}^{G H, A B} C_{K L}^{C D, E F}+C_{E F}^{C D, G H} C_{K L}^{A B, G H}=0 .
$$

This Lie 3-algebra is also accompanied by a metric $h^{A B}=\operatorname{Tr}\left(T^{A} T^{B}\right)$ which is used to raise and lower the indices.

Now the scalar superfields $X^{I}$ and $X^{\dagger}$ are suitably contracted with the generators of this Lie 3 -algebra, $X^{I}=X^{A I} T_{A}$, and $X^{I \dagger}=X^{I A \dagger} T_{A}$, and transform under infinitesimal gauge transformations as

$$
\begin{aligned}
\delta X^{I A} & =i \Lambda^{A B} X_{B}^{I}, \\
\delta X^{I A \dagger} & =-i X_{B}^{I \dagger} \Lambda^{A B} .
\end{aligned}
$$

It is convenient to define $\Lambda=\Lambda^{A B} T_{A} T_{B}$ and the following super-covariant derivatives of these superfields

$$
\begin{aligned}
\nabla_{a} X_{A}^{I} & =D_{a} X_{A}-i f_{A}^{B C D} \Gamma_{a B C} X_{D}^{I}, \\
\nabla_{a} X_{A}^{I \dagger} & =D_{a} X_{A}^{I \dagger}+i f_{A}^{B C D} X_{D}^{I \dagger} \Gamma^{a B C},
\end{aligned}
$$

where

$$
D_{a}=\partial_{a}+\left(\gamma^{\mu} \partial_{\mu}\right)_{a}^{b} \theta_{b} .
$$

It is also convenient to define $\Gamma_{a}$ as a matrix valued spinor superfield suitable contracted with generators of a Lie 3-algebra, $\Gamma_{a}=\Gamma_{a}^{A B} T_{A} T_{B}$. This matrix valued spinor superfield transforms under gauge transformations as

$$
\delta \Gamma_{a}=\nabla_{a} \Lambda,
$$

where

$$
\nabla_{a} \Lambda=\left[D_{a} \Lambda_{A B}+C_{A B}^{C D, E F} \Gamma_{C D a} \Lambda_{E F}\right] T^{A} T^{B} .
$$

In fact, we will define the covariant derivative on $\Gamma_{a}^{A B}$ as follows

$$
\left(\nabla_{a} \Gamma_{b}\right)_{A B}=D_{a} \Gamma_{b A B}+C_{A B}^{C D, E F} \Gamma_{C D a} \Gamma_{b E F} .
$$

It is useful to define ordinary gauge covariant derivatives for component of matter and gauge superfields. Thus, if $a_{i}^{A}$ and $\bar{a}_{i}^{A}$ are components of the matter fields then the ordinary gauge covariant derivatives for them are given by

$$
\begin{aligned}
& \mathcal{D}_{\mu} a_{i}^{A}=\partial_{\mu} a_{i}^{A}-i f_{D}^{A B C} A_{\mu B C} a_{i}^{D}, \\
& \mathcal{D}_{\mu} \bar{a}_{i}^{A}=\partial_{\mu} \bar{a}_{i}^{A}+i f_{D}^{A B C} \bar{a}_{i}^{D} A_{\mu B C} .
\end{aligned}
$$

Similarly, the ordinary gauge covariant derivative for the component fields $e_{i}^{A B}$ of the gauge superfield is given by

$$
\mathcal{D}_{\mu} e_{i A B}=\partial_{\mu} e_{i A B}+C_{A B}^{C D, E F} A_{\mu C D} e_{i E F} .
$$

Here $A_{\mu}^{A B}$ is the ordinary gauge connection which is given by

$$
A^{\mu A B}=-\frac{1}{2}\left[\left(\nabla^{a}\left(\gamma^{\mu}\right)_{a}^{b} \Gamma_{b}\right)^{A B}\right]_{\mid},
$$

where ${ }^{\prime} \mid$ ' means that the quantity is evaluated at $\theta_{a}=0$. 
Now we can calculate the following

$$
\begin{aligned}
\left(\left\{\nabla_{a}, \nabla_{b}\right\} X^{I}\right)_{A}= & \left(\nabla_{a} \nabla_{b} X^{I}\right)_{A}+\left(\nabla_{b} \nabla_{a} X^{I}\right)_{A} \\
= & \left(D_{a} \delta_{A}^{D}-i f_{A}^{B C D} \Gamma_{a C D}\right) \\
& \times\left(D_{b} \delta_{D}^{G}-i f_{D}^{E F G} \Gamma_{b E F}\right) X_{G}^{I} \\
& -\left(D_{b} \delta_{A}^{D}-i f_{A}^{B C D} \Gamma_{b C D}\right) \\
& \times\left(D_{a} \delta_{D}^{G}-i f_{D}^{E F G} \Gamma_{a E F}\right) X_{G}^{I} \\
= & 2\left(\gamma_{a b}^{\mu} \mathcal{D}_{\mu} X^{I}\right)_{A},
\end{aligned}
$$

and

$$
\begin{aligned}
\left(\left\{\nabla_{a}, \nabla_{b}\right\} X^{I \dagger}\right)_{A}= & \left(\nabla_{a} \nabla_{b} X^{I}\right)_{A}+\left(\nabla_{b} \nabla_{a} X^{I \dagger}\right)_{A} \\
= & \left(D_{a} \delta_{A}^{D}+i f_{A}^{B C D} \Gamma_{a C D}\right) \\
& \times\left(D_{b} \delta_{D}^{G}+i f_{D}^{E F G} \Gamma_{b E F}\right) X_{G}^{I \dagger} \\
& -\left(D_{b} \delta_{A}^{D}+i f_{A}^{B C D} \Gamma_{b C D}\right) \\
& \times\left(D_{a} \delta_{D}^{G}+i f_{D}^{E F G} \Gamma_{a E F}\right) X_{G}^{I \dagger} \\
= & 2\left(\gamma_{a b}^{\mu} \mathcal{D}_{\mu} X^{I \dagger}\right)_{A} .
\end{aligned}
$$

We also have

$$
\begin{aligned}
\left(\left\{\nabla_{a}, \nabla_{b}\right\} \Gamma_{c}\right)_{A B}= & \left(\nabla_{a} \nabla_{b} \Gamma_{c}\right)_{A B}+\left(\nabla_{b} \nabla_{a} \Gamma_{c}\right)_{A B} \\
= & \left(D_{a} \delta_{E}^{A} \delta_{F}^{E}+C_{A B}^{C D, E F} \Gamma_{a C D}\right) \\
& \times\left(D_{b} \delta_{E}^{L} \delta_{F}^{M}+C_{E F}^{G H, L M} \Gamma_{b G H}\right) \Gamma_{c L M} \\
& -\left(D_{b} \delta_{E}^{A} \delta_{F}^{E}+C_{A B}^{C D, E F} \Gamma_{b C D}\right) \\
& \times\left(D_{a} \delta_{E}^{L} \delta_{F}^{M}+C_{E F}^{G H, L M} \Gamma_{a G H}\right) \Gamma_{c L M} \\
= & 2\left(\gamma_{a b}^{\mu} \mathcal{D}_{\mu} \Gamma_{c}\right)_{A B} .
\end{aligned}
$$

Using $X_{A}^{I} T^{A}=X^{I}, X_{A}^{I \dagger} T^{A}=X^{I \dagger}$ and $\Gamma_{c A B} T^{A} T^{B}=\Gamma_{c}$, we have

$$
\begin{aligned}
\left\{\nabla_{a}, \nabla_{b}\right\} X^{I} & =2 \gamma_{a b}^{\mu} \mathcal{D}_{\mu} X^{I}, \\
\left\{\nabla_{a}, \nabla_{b}\right\} X^{I \dagger} & =2 \gamma_{a b}^{\mu} \mathcal{D}_{\mu} X^{I \dagger}, \\
\left\{\nabla_{a}, \nabla_{b}\right\} \Gamma_{c} & =2 \gamma_{a b}^{\mu} \mathcal{D}_{\mu} \Gamma_{c} .
\end{aligned}
$$

Now we can write $\left(\nabla_{a} \nabla_{b} X^{I}\right)_{A},\left(\nabla_{a} \nabla_{b} X^{I \dagger}\right)_{A}$ and $\left(\nabla_{a} \nabla_{b} \Gamma_{c}\right)_{A B}$ as a half of the sum of there commutator with its anticommutator,

$$
\begin{aligned}
\left(\nabla_{a} \nabla_{b} X^{I}\right)_{A} & =\frac{1}{2}\left(\left\{\nabla_{a}, \nabla_{b}\right\} X^{I}\right)_{A}+\frac{1}{2}\left(\left[\nabla_{a}, \nabla_{b}\right] X^{I}\right)_{A}, \\
\left(\nabla_{a} \nabla_{b} X^{I \dagger}\right)_{A} & =\frac{1}{2}\left(\left\{\nabla_{a}, \nabla_{b}\right\} X^{I \dagger}\right)_{A}+\frac{1}{2}\left(\left[\nabla_{a}, \nabla_{b}\right] X^{I \dagger}\right)_{A}, \\
\left(\nabla_{a} \nabla_{b} \Gamma_{c}\right)_{A B} & =\frac{1}{2}\left(\left\{\nabla_{a}, \nabla_{b}\right\} \Gamma_{c}\right)_{A B}+\frac{1}{2}\left(\left[\nabla_{a}, \nabla_{b}\right] \Gamma_{c}\right)_{A B} .
\end{aligned}
$$

However, for $\mathcal{N}=1$ superfields in three dimensions the indices ' $a$ ' are twodimensional. Thus, the anticommutator $\left(\left[\nabla_{a}, \nabla_{b}\right] X^{I}\right)_{A},\left(\left[\nabla_{a}, \nabla_{b}\right] X^{I \dagger}\right)_{A}$, and $\left(\left[\nabla_{a}, \nabla_{b}\right] \Gamma_{c}\right)_{A B}$ must be proportional to anti-symmetric tensors some $\left(C_{a b} X^{I}\right)_{A}$, 
$\left(C_{a b} X^{I \dagger}\right)_{A}$, and $\left(C_{a b} \Gamma_{c}\right)_{A B}$, respectively. So, we find

$$
\begin{gathered}
\left(\nabla_{a} \nabla_{b} X^{I}\right)_{A}=\left(\left(\gamma_{a b}^{\mu} \mathcal{D}_{\mu}-C_{a b} \nabla^{2}\right) X^{I}\right)_{A}, \\
\left(\nabla_{a} \nabla_{b} X^{I \dagger}\right)_{A}=\left(\left(\gamma_{a b}^{\mu} \mathcal{D}_{\mu}-C_{a b} \nabla^{2}\right) X^{I \dagger}\right)_{A}, \\
\left(\nabla_{a} \nabla_{b} \Gamma_{c}\right)_{A B}=\left(\left(\gamma_{a b}^{\mu} \mathcal{D}_{\mu}-C_{a b} \nabla^{2}\right) \Gamma_{c}\right)_{A B} .
\end{gathered}
$$

Now using [25]

$$
D_{a} D_{b} D_{c}=\frac{1}{2} D_{a}\left\{D_{b}, D_{c}\right\}-\frac{1}{2} D_{b}\left\{D_{a}, D_{c}\right\}+\frac{1}{2} D_{c}\left\{D_{a}, D_{b}\right\},
$$

along with Eqs. (21) and (41), we get,

$$
\begin{aligned}
\left(\nabla_{a} \nabla_{b} \nabla_{c} X^{I}\right)_{A}= & \frac{1}{2}\left(\nabla_{a}\left\{\nabla_{b}, \nabla_{c}\right\} X^{I}\right)_{A}-\frac{1}{2}\left(\nabla_{b}\left\{\nabla_{a}, \nabla_{c}\right\} X^{I}\right)_{A} \\
& +\frac{1}{2}\left(\nabla_{c}\left\{\nabla_{a}, \nabla_{b}\right\} X^{I}\right)_{A}, \\
\left(\nabla_{a} \nabla_{b} \nabla_{c} X^{I \dagger}\right)_{A}= & \frac{1}{2}\left(\nabla_{a}\left\{\nabla_{b}, \nabla_{c}\right\} X^{I \dagger}\right)_{A}-\frac{1}{2}\left(\nabla_{b}\left\{\nabla_{a}, \nabla_{c}\right\} X^{I \dagger}\right)_{A} \\
& +\frac{1}{2}\left(\nabla_{c}\left\{\nabla_{a}, \nabla_{b}\right\} X^{I \dagger}\right)_{A}, \\
\left(\nabla_{a} \nabla_{b} \nabla_{c} \Gamma_{d}\right)_{A B}= & \frac{1}{2}\left(\nabla_{a}\left\{\nabla_{b}, \nabla_{c}\right\} \Gamma_{d}\right)_{A B}-\frac{1}{2}\left(\nabla_{b}\left\{\nabla_{a}, \nabla_{c}\right\} \Gamma_{d}\right)_{A B} \\
& +\frac{1}{2}\left(\nabla_{c}\left\{\nabla_{a}, \nabla_{b}\right\} \Gamma_{d}\right)_{A B} .
\end{aligned}
$$

Thus, we get

$$
\left(\nabla^{a} \nabla_{b} \nabla_{a} X^{I}\right)_{A}=\left(\nabla^{a} \nabla_{b} \nabla_{a} X^{I \dagger}\right)_{A}=\left(\left(\nabla^{a} \nabla_{b} \nabla_{a} \Gamma_{c}\right)_{A B}=0,\right.
$$

and

$$
\begin{gathered}
\left(\nabla^{2} \nabla_{a} X^{I}\right)_{A}=\left(\left(\gamma^{\mu} \nabla\right)_{a} \mathcal{D}_{\mu} X^{I}\right)_{A}, \\
\left(\nabla^{2} \nabla_{a} X^{I \dagger}\right)_{A}=\left(\left(\gamma^{\mu} \nabla\right)_{a} \mathcal{D}_{\mu} X^{I \dagger}\right)_{A}, \\
\left(\nabla^{2} \nabla_{a} \Gamma_{c}\right)_{A B}=\left(\left(\gamma^{\mu} \nabla\right)_{a} \mathcal{D}_{\mu} \Gamma_{c}\right)_{A B} .
\end{gathered}
$$

\section{Boundary Super-Covariant Derivatives}

In the previous section we analysed some properties of super-covariant derivatives for a gauge theory where the gauge symmetry is generated by a Lie 3algebra. In this section we will analyse the effect of having a boundary on these super-covariant derivatives. We start by having a boundary at fixed $x^{3}$. Thus our indices for the coordinates will splits as $\mu=(m, 3)$. We will denoted the induced value of the fields $X, X^{\dagger}, \Gamma_{a}, \Lambda$ on the boundary by $X^{\prime}, X^{\dagger^{\prime}}, \Gamma_{a}^{\prime}, \Lambda^{\prime}$ and the induced value of the super-derivative $D_{a}$ and the super-covariant derivative $\nabla_{a}$ on the boundary will be denoted by $D_{a}^{\prime}$ and $\nabla_{a}^{\prime}$, respectively. This boundary super-derivative $D_{a}^{\prime}$ is obtained by neglecting $\gamma^{3} \partial_{3}$ contributions in $D_{a}$,

$$
D_{a}^{\prime}=\partial_{a}+\left(\gamma^{m} \partial_{m}\right)_{a}^{b} \theta_{b}
$$


The boundary super-covariant derivative $\nabla_{a}^{\prime}$ can thus be written as

$$
\begin{aligned}
\left(\nabla_{a}^{\prime} X^{I^{\prime}}\right)_{A} & =D_{a}^{\prime} X_{A}^{I^{\prime}}-i f_{A}^{B C D} \Gamma_{B C a}^{\prime} X_{D}^{I^{\prime}}, \\
\left(\nabla_{a}^{\prime} X^{I \dagger^{\prime}}\right)_{A} & =D_{a}^{\prime} X^{I^{\prime}{ }_{A}}+i f_{A}^{B C D} X_{D_{D}{ }^{\prime} \Gamma_{B C a}^{\prime},} \\
\left(\nabla_{a}^{\prime} \Gamma_{b}^{\prime}\right)_{A B} & =D_{a}^{\prime} \Gamma_{b A B}^{\prime}+C_{A B}^{C D, E F} \Gamma_{C D a}^{\prime} \Gamma_{b E F}^{\prime}, \\
\left(\nabla_{a}^{\prime} \Lambda^{\prime}\right)_{A B} & =D_{a}^{\prime} \Lambda_{A B}^{\prime}+C_{A B}^{C D, E F} \Gamma_{C D a}^{\prime} \Lambda_{E F}^{\prime} .
\end{aligned}
$$

In fact, this notation will be used to denote all boundary quantities along with the induced value of any bulk quantity on the boundary. Thus, this convention will be followed even for component fields of superfields. Now we define projection operators $P_{ \pm}$as

$$
\left(P_{ \pm}\right)_{a b}=\frac{1}{2}\left(C_{a b} \pm\left(\gamma^{3}\right)_{a b}\right) .
$$

These projection operators can be used to project the super-covariant derivative $\nabla_{a}$ as, $\nabla_{ \pm b}=\left(P_{ \pm}\right)_{b}^{a} \nabla_{a}$, and $\nabla_{ \pm b}^{\prime}$ as, $\nabla_{ \pm b}^{\prime}=\left(P_{ \pm}\right)_{b}^{a} \nabla_{a}^{\prime}$, where $\nabla_{ \pm a}^{\prime}$ is the induced value of $\nabla_{ \pm a}$ on the boundary. Similarly, for the ordinary gauge covariant derivatives we have $\left(P_{ \pm} \gamma^{m}\right)_{a b} \mathcal{D}_{m}=\left(\gamma^{ \pm}\right)_{a b} \mathcal{D}_{ \pm}$, where $\gamma^{ \pm}=\gamma^{0} \pm \gamma^{1}$ and $\mathcal{D}_{ \pm}=$ $\frac{1}{2}\left(\mathcal{D}_{0} \pm \mathcal{D}_{1}\right)$. Now we can calculate the projected values of the super-covariant derivative acting on $X_{A}^{I}$

$$
\begin{aligned}
\left(\nabla_{+a} \nabla_{+b} X^{I}\right)_{A} & =\left(\left(P_{+}\right)_{a}^{c}\left(P_{+}\right)_{b}^{d}\left(\nabla_{c} \nabla_{d} X^{I}\right)_{A}\right. \\
& =-\left(\left(\gamma^{+}\right)_{a b} \mathcal{D}_{+} X^{I}\right)_{A}, \\
\left(\nabla_{-a} \nabla_{-b} X^{I}\right)_{A} & =\left(\left(P_{-}\right)_{a}^{c}\left(P_{-}\right)_{b}^{d}\left(\nabla_{c} \nabla_{d} X^{I}\right)_{A}\right. \\
& =-\left(\left(\gamma^{-}\right)_{a b} \mathcal{D}_{-} X^{I}\right)_{A}, \\
\left(\nabla_{-a} \nabla_{+b} X^{I}\right)_{A} & =\left(\left(P_{-}\right)_{a}^{c}\left(P_{+}\right)_{b}^{d}\left(\nabla_{c} \nabla_{d} X^{I}\right)_{A}\right. \\
& =-\left(\left(P_{-}\right)_{a b}\left(\mathcal{D}_{3}+\nabla^{2}\right) X^{I}\right)_{A}, \\
\left(\nabla_{+a} \nabla_{-b} X^{I}\right)_{A} & =\left(\left(P_{+}\right)_{a}^{c}\left(P_{-}\right)_{b}^{d}\left(\nabla_{c} \nabla_{d} X^{I}\right)_{A}\right. \\
& =-\left(\left(P_{+}\right)_{a b}\left(-\mathcal{D}_{3}+\nabla^{2}\right) X^{I}\right)_{A} .
\end{aligned}
$$

Similarly, we can calculate the projected values of the super-covariant derivative acting on $X_{A}^{I \dagger}$

$$
\begin{aligned}
\left(\nabla_{+a} \nabla_{+b} X^{I \dagger}\right)_{A} & =\left(\left(P_{+}\right)_{a}^{c}\left(P_{+}\right)_{b}^{d}\left(\nabla_{c} \nabla_{d} X^{I \dagger}\right)_{A}\right. \\
& =-\left(\left(\gamma^{+}\right)_{a b} \mathcal{D}_{+} X^{I \dagger}\right)_{A}, \\
\left(\nabla_{-a} \nabla_{-b} X^{I \dagger}\right)_{A} & =\left(\left(P_{-}\right)_{a}^{c}\left(P_{-}\right)_{b}^{d}\left(\nabla_{c} \nabla_{d} X^{I \dagger}\right)_{A}\right. \\
& =-\left(\left(\gamma^{-}\right)_{a b} \mathcal{D}_{-} X^{I \dagger}\right)_{A}, \\
\left(\nabla_{-a} \nabla_{+b} X^{I \dagger}\right)_{A} & =\left(\left(P_{-}\right)_{a}^{c}\left(P_{+}\right)_{b}^{d}\left(\nabla_{c} \nabla_{d} X^{I \dagger}\right)_{A}\right. \\
& =-\left(\left(P_{-}\right)_{a b}\left(\mathcal{D}_{3}+\nabla^{2}\right) X^{I \dagger}\right)_{A}, \\
\left(\nabla_{+a} \nabla_{-b} X^{I \dagger}\right)_{A} & =\left(\left(P_{+}\right)_{a}^{c}\left(P_{-}\right)_{b}^{d}\left(\nabla_{c} \nabla_{d} X^{I \dagger}\right)_{A}\right. \\
& =-\left(\left(P_{+}\right)_{a b}\left(-\mathcal{D}_{3}+\nabla^{2}\right) X^{I \dagger}\right)_{A} .
\end{aligned}
$$

Finally we can calculate the projected value of the super-covariant derivative acting on $\Gamma_{c}^{A B}$

$$
\left(\nabla_{+a} \nabla_{+b} \Gamma_{c}\right)_{A B}=\left(\left(P_{+}\right)_{a}^{c}\left(P_{+}\right)_{b}^{d}\left(\nabla_{c} \nabla_{d} \Gamma_{c}\right)_{A}\right.
$$




$$
\begin{aligned}
& =-\left(\left(\gamma^{+}\right)_{a b} \mathcal{D}_{+} X^{I}\right)_{A B}, \\
\left(\nabla_{-a} \nabla_{-b} \Gamma_{c}\right)_{A B} & =\left(\left(P_{-}\right)_{a}^{c}\left(P_{-}\right)_{b}^{d}\left(\nabla_{c} \nabla_{d} \Gamma_{c}\right)_{A}\right. \\
& =-\left(\left(\gamma^{-}\right)_{a b} \mathcal{D}_{-} X^{I}\right)_{A B}, \\
\left(\nabla_{-a} \nabla_{+b} \Gamma_{c}\right)_{A B} & =\left(\left(P_{-}\right)_{a}^{c}\left(P_{+}\right)_{b}^{d}\left(\nabla_{c} \nabla_{d} \Gamma_{c}\right)_{A}\right. \\
& =-\left(\left(P_{-}\right)_{a b}\left(\mathcal{D}_{3}+\nabla^{2}\right) X^{I}\right)_{A B}, \\
\left(\nabla_{+a} \nabla_{-b} \Gamma_{c}\right)_{A B} & =\left(\left(P_{+}\right)_{a}^{c}\left(P_{-}\right)_{b}^{d}\left(\nabla_{c} \nabla_{d} \Gamma_{c}\right)_{A}\right. \\
& =-\left(\left(P_{+}\right)_{a b}\left(-\mathcal{D}_{3}+\nabla^{2}\right) X^{I}\right)_{A B} .
\end{aligned}
$$

Suitably contracting the fields with generators of the Lie 3 algebra as $X_{A}^{I} T^{A}=$ $X^{I}, X_{A}^{I \dagger} T^{A}=X^{I \dagger}$ and $\Gamma_{c A B} T^{A} T^{B}=\Gamma_{c}$, we get the following algebra

$$
\begin{array}{cc}
\left\{\nabla_{+a}, \nabla_{+b}\right\} X^{I}=-2\left(\gamma^{+}\right)_{a b} \mathcal{D}_{+} X^{I}, & \left\{\nabla_{-a}, \nabla_{-b}\right\} X^{I}=-2\left(\gamma^{-}\right)_{a b} \mathcal{D}_{-} X^{I}, \\
\left\{\nabla_{-a}, \nabla_{+b}\right\} X^{I}=-2\left(P_{-}\right)_{a b} \mathcal{D}_{3} X^{I}, & \left\{\nabla_{+a}, \nabla_{+b}\right\} X^{I \dagger}=-2\left(\gamma^{+}\right)_{a b} \mathcal{D}_{+} X^{I \dagger}, \\
\left\{\nabla_{-a}, \nabla_{-b}\right\} X^{I \dagger}=-2\left(\gamma^{-}\right)_{a b} \mathcal{D}_{-} X^{I \dagger}, & \left\{\nabla_{-a}, \nabla_{+b}\right\} X^{I \dagger}=-2\left(P_{-}\right)_{a b} \mathcal{D}_{3} X^{I \dagger}, \\
\left\{\nabla_{+a}, \nabla_{+b}\right\} \Gamma_{c}=-2\left(\gamma^{+}\right)_{a b} \mathcal{D}_{+} \Gamma_{c}, & \left\{\nabla_{-a}, \nabla_{-b}\right\} \Gamma_{c}=-2\left(\gamma^{-}\right)_{a b} \mathcal{D}_{-} \Gamma_{c}, \\
\left\{\nabla_{-a}, \nabla_{+b}\right\} \Gamma_{c}=-2\left(P_{-}\right)_{a b} \mathcal{D}_{3} \Gamma_{c} . &
\end{array}
$$

Now we have effectively decomposed $\mathcal{N}=1$ superfields in three dimensions into $\mathcal{N}=(1,0)$ in two dimensions. The generators of the $\mathcal{N}=1$ supersymmetry in three dimensions is

$$
Q_{a}=\partial_{a}-\left(\gamma^{\mu} \partial_{\mu}\right)_{a}^{b} \theta_{b} .
$$

This generator of $\mathcal{N}=1$ supersymmetry in three dimensions splits into $Q_{ \pm a}=$ $P_{ \pm a}^{b} Q_{b}$ in two dimensions,

$$
\epsilon^{a} Q_{a}=\epsilon^{a-} Q_{-a}+\epsilon^{a+} Q_{+a},
$$

where $\epsilon_{a \pm}=P_{ \pm a}^{b} \epsilon_{b}$. It can be shown that these generators of the supersymmetry in presence of a boundary satisfy

$$
\begin{aligned}
& \left\{Q_{+a}, Q_{+b}\right\}=2\left(\gamma^{+}\right)_{a b} \partial_{+}, \quad\left\{Q_{-a}, Q_{-b}\right\}=2\left(\gamma^{-}\right)_{a b} \partial_{-}, \\
& \left\{Q_{-a}, Q_{+b}\right\}=2\left(P_{-}\right)_{a b} \partial_{3},
\end{aligned}
$$

where $\partial_{ \pm}=\frac{1}{2}\left(\partial_{0} \pm \partial_{1}\right)$. It may be noted in absence of a boundary term this algebra is actually the algebra of two copies of $\mathcal{N}=1$ supersymmetric theories in two dimensions.

\section{Supersymmetry in Presence of a Boundary}

To analyse the boundary effects in the BLG theory we first construct the BLG theory on manifolds without boundaries. The Lagrangian for the BLG theory in $\mathcal{N}=1$ superspace formalism is given by

$$
\mathcal{L}_{B L G}=\mathcal{L}_{C S}+\mathcal{L}_{K E}+\mathcal{L}_{V},
$$

where $\mathcal{L}_{C S}$ is the Chern-Simon term, $\mathcal{L}_{K E}$ is the the kinetic energy term and $\mathcal{L}_{V}$ is the potential energy part term of the BLG theory. In our notations a trace 
over the Lie 3-algebra will be assumed in the Lagrangian of the BLG theory. Now the Chern-Simon term is given by

$$
\mathcal{L}_{C S}=-\frac{k}{4 \pi} \nabla^{2}\left[f^{A B C D} \Gamma_{A B}^{a} \Omega_{a C D}\right] \mid
$$

where

$$
\begin{aligned}
\Omega_{a A B}= & \omega_{a A B}-\frac{1}{3} C_{A B}^{C D, E F}\left[\Gamma^{b C D}, \Gamma_{a b E F}\right] \\
\omega_{a A B}= & \frac{1}{2} D^{b} D_{a} \Gamma_{b A B}-i C_{A B}^{C D, E F}\left[\Gamma_{C D}^{b}, D_{b} \Gamma_{a E F}\right] \\
& -\frac{1}{3} C_{A B}^{C D, E F} C_{E F}^{G H, I J}\left[\Gamma_{C D}^{b},\left\{\Gamma_{b G H}, \Gamma_{a I J}\right\}\right] \\
\Gamma_{a b A B}= & -\frac{i}{2}\left[D_{(a} \Gamma_{b) A B}-2 i C_{A B}^{C D, E F}\left\{\Gamma_{a C D}, \Gamma_{b E F}\right\}\right] .
\end{aligned}
$$

The covariant divergence of $\omega_{a A B}$ vanishes

$$
\begin{aligned}
\left(\nabla^{a} \omega_{a}\right)_{A B}= & {\left[D^{a} \delta_{A}^{E} \delta_{B}^{F}+C_{A B}^{C D, E F} \Gamma_{C D}^{a}\right] \omega_{a E F} } \\
= & \frac{1}{2} \delta_{A}^{E} \delta_{B}^{F} D^{a} D^{b} D_{a} \Gamma_{b E F}-i C_{E F}^{C D, L M} \delta_{A}^{E} \delta_{B}^{F} D^{a}\left[\Gamma_{C D}^{b}, D_{b} \Gamma_{a L M}\right] \\
& -\frac{1}{3} C_{E F}^{C D, L M} C_{L M}^{G H, I J} \delta_{A}^{E} \delta_{B}^{F} D^{a}\left[\Gamma_{C D}^{b},\left\{\Gamma_{b G H}, \Gamma_{a I J}\right\}\right] \\
& -i C_{A B}^{C D, E F} C_{E F}^{I J, L M} \Gamma_{C D}^{a}\left[\Gamma_{I J}^{b}, D_{b} \Gamma_{a L M}\right] \\
& -\frac{1}{3} C_{E F}^{C D, L M} C_{L M}^{G H, I J} C_{A B}^{S T, E F} \Gamma_{C D}^{a}\left[\Gamma_{S T}^{b},\left\{\Gamma_{b G H}, \Gamma_{a I J}\right\}\right] \\
& +\frac{1}{2} C_{A B}^{C D, E F} \Gamma_{C D}^{a} D^{b} D_{a} \Gamma_{b E F} \\
= & 0,
\end{aligned}
$$

here we have used $D^{a} D_{b} D_{a}=0,\left[25\right.$. Now the components of $\Gamma_{a}^{A B}$ are given by

$$
\begin{aligned}
\chi_{a}^{A B}=\left[\Gamma_{a}^{A B}\right]_{\mid}, & A^{A B}=-\frac{1}{2}\left[\left(\nabla^{a} \Gamma_{a}\right)^{A B}\right]_{\mid}, \\
A^{\mu A B}=-\frac{1}{2}\left[\left(\nabla^{a}\left(\gamma^{\mu}\right)_{a}^{b} \Gamma_{b}\right)^{A B}\right]_{\mid}, & E_{a}^{A B}=-\left[\left(\nabla^{b} \nabla_{a} \Gamma_{b}\right)^{A B}\right]_{\mid},
\end{aligned}
$$

and so the components of the $\omega_{a}^{A B}$ can now be written as

$$
\begin{aligned}
{\left[\left(\nabla^{a}\left(\gamma^{\mu}\right)_{a}^{b} \omega_{b}\right)^{A B}\right]_{\mid}=\epsilon^{\mu \nu \rho} F_{\nu \rho}^{A B}, } & {\left[\left(\nabla^{a} \omega_{a}\right)^{A B}\right]_{\mid}=0, } \\
-\left[\left(\nabla^{b} \nabla_{a} \omega_{b}\right)^{A B}\right]_{\mid}=2\left(\left(\gamma^{\mu} \mathcal{D}_{\mu}\right)_{a}^{b} E_{b}\right)^{A B}, & {\left[\omega_{a}^{A B}\right]_{\mid}=E_{a}^{A B}, }
\end{aligned}
$$

where $\epsilon_{\mu \nu \rho}$ is an anti-symmetric tensor. Thus, the component form for ChernSimons term can be written as

$$
\begin{aligned}
\mathcal{L}_{C S}= & \frac{k}{4 \pi}\left[\epsilon^{\mu \nu \rho}\left(f^{A B C D} A_{\mu A B} \partial_{\nu} A^{\rho C D}+\frac{i}{3} C_{A B}^{C D, E F} A_{\mu}^{A B} A_{\nu C D} A_{\rho E F}\right)\right. \\
& +f^{A B C D}\left(E_{A B}^{a} E_{a C D}+\left(\mathcal{D}_{\mu} \chi^{a}\left(\gamma^{\mu}\right)_{a}^{b}\right)_{A B} E_{b C D}\right. \\
& \left.\left.+\left(\chi^{a}\left(\gamma^{\mu}\right)_{a}^{b}\right)_{A B}\left(\mathcal{D}_{\mu} E_{b}\right)_{C D}\right)\right] .
\end{aligned}
$$


The Kinetic energy term for the BLG theory can be written as

$$
\mathcal{L}_{K E}=-\frac{1}{4} \nabla^{2}\left[\left(\nabla^{a} X^{I}\right)^{A}\left(\nabla_{a} X_{I}^{\dagger}\right)_{A}\right]_{\mid},
$$

and the potential term for the BLG theory can be written as

$$
\mathcal{L}_{V}=-\frac{2 \pi}{k} \nabla^{2}\left[\epsilon_{I J K L} f^{A B C D} X_{A}^{I} X_{B}^{K \dagger} X_{C}^{J} X_{D}^{L \dagger}\right]_{\mid} .
$$

This theory is manifestly invariant under $\mathcal{N}=1$ superspace transformations, even thought in reality it has $\mathcal{N}=8$ supersymmetry. Thus, we have

$$
\begin{aligned}
\delta_{S} \mathcal{L}_{B L G} & =\epsilon^{a} Q_{a} \mathcal{L}_{B L G} \\
& =\mathcal{D}_{\mu}\left[\Phi\left(\gamma^{\mu}, \Gamma, X^{I}, X^{I^{\dagger}}\right)\right]_{\mid} .
\end{aligned}
$$

Now in absence of a boundary, we have

$$
\delta_{S} \mathcal{L}_{B L G}=0
$$

In fact, the supersymmetric variation of any superfield theory written in the $\mathcal{N}=1$ superspace formalism gives rise to a total derivative term [25]. Thus, in absence of a boundary this term vanishes and the theory has manifest $\mathcal{N}=1$ supersymmetry.

Now if the full finite gauge transformation of the superfield $\Gamma_{a}=\Gamma_{a}^{A B} T_{A} T_{B}$ is given by

$$
\Gamma_{a} \rightarrow i u \nabla_{a} u^{-1}
$$

where

$$
u=\exp \left(i \Lambda^{A B} T_{A} T_{B}\right),
$$

then the gauge transformation of the superfield $\omega_{a}=\omega_{a}^{A B} T_{A} T_{B}$ will be given by

$$
\omega_{a} \rightarrow u \omega_{a} u^{-1} \text {. }
$$

Similarly, the finite gauge transformations of $X^{I}$ and $X^{\dagger I}$ will be given by

$$
\begin{aligned}
X^{I A} & \rightarrow u X^{I}, \\
X^{I A \dagger} & \rightarrow X_{B}^{I \dagger} u^{-1} .
\end{aligned}
$$

Thus, under infinitesimal gauge transformations the Lagrangian for the $\mathcal{N}=1$ non-Abelian Chern-Simons theory will transforms as

$$
\begin{aligned}
\delta \mathcal{L}_{C S} & =-\frac{k}{4 \pi} \nabla^{2}\left[f^{A B C D} D^{a} \Lambda_{A B} \omega_{a C D}+C_{A B}^{C D, E F} \Gamma_{C D}^{a} \Lambda_{E F} \omega_{a}^{A B}\right]_{\mid} \\
& =-\frac{k}{4 \pi} \nabla^{2}\left[f^{A B C D}\left(\nabla^{a} \Lambda\right)_{A B} \omega_{a C D}\right]_{\mid} .
\end{aligned}
$$

As the covariant derivative of $\omega_{a A B}$ vanishes, Eq. (48), we get

$$
\delta \mathcal{L}_{C S}=-\frac{k}{4 \pi} \nabla^{2} \nabla^{a}\left[f^{A B C D} \Lambda_{A B} \omega_{a C D}\right]_{1}
$$

Now using Eq. 23, we get

$$
\delta \mathcal{L}_{C S}=-\frac{k}{4 \pi}\left(\gamma^{\mu} \mathcal{D}_{\mu} \nabla\right)^{a}\left[f^{A B C D} \Lambda_{A B} \omega_{a C D}\right]_{\mid} .
$$


This is a total derivative, so on a manifold without a boundary, we have

$$
\delta \mathcal{L}_{C S}=0 .
$$

Thus, the $\mathcal{N}=1$ non-Abelian Chern-Simons theory is invariant under these gauge transformations on a manifold without a boundary.

Now after analysing the BLG theory on manifolds without boundaries, we will analyse the effect of the boundary on the manifest $\mathcal{N}=1$ supersymmetric of the theory. As the supersymmetric variation of the BLG theory gives rise to a total derivative term, we have

$$
\begin{aligned}
\delta_{S} \mathcal{L}_{B L G} & =\epsilon^{a} Q_{a} \mathcal{L}_{B L G} \\
& =\mathcal{D}_{\mu}\left[\Phi\left(\gamma^{\mu}, \Gamma, X^{I}, X^{I \dagger}\right)\right]_{\mid} \\
& \sim \mathcal{D}_{3}\left[\Phi\left(\gamma^{3}, \Gamma, X^{I}, X^{I \dagger}\right)\right]_{\mid} .
\end{aligned}
$$

where $\sim$ indicates that we have neglected the total derivative contribution along directions other than $x^{3}$, as they will not contribute. So, in presence of a boundary, this supersymmetric variation of the BLG theory will gives rise to a boundary term,

$$
\delta_{S} \mathcal{L}_{B L G}=\left[\Phi^{\prime}\left(\gamma^{3}, \Gamma^{\prime}, X^{I^{\prime}}, X^{I^{\dagger^{\prime}}}\right)\right]_{\mid} .
$$

Thus, the manifest $\mathcal{N}=1$ supersymmetry will be broken in presence of a boundary.

The supersymmetry of the theory can be restored by adding a boundary term whose supersymmetric variation exactly cancels the supersymmetric variation of the original theory. This has already been done for Abelian Chern-Simons theories 25, 26. These results can be generalised to the present case. The term that needs to be added to the original BLG theory can be written as

$$
\mathcal{L}_{b, B L G}=\mathcal{L}_{b, C S}+\mathcal{L}_{b, K E}+\mathcal{L}_{b, V},
$$

where $\mathcal{L}_{b, C S}$ is the boundary term corresponding to the Chern-Simon term, $\mathcal{L}_{b, K E}$ is the boundary term corresponding to the kinetic energy term and $\mathcal{L}_{b, V}$ is the boundary term corresponding to the potential energy part term. Now $\mathcal{L}_{b, C S}$ is given by

$$
\mathcal{L}_{b, C S}=\frac{k}{4 \pi} \mathcal{D}_{3}\left[f^{A B C D} \Gamma_{A B}^{a} \Omega_{a C D}\right]_{\mid},
$$

$\mathcal{L}_{b, K E}$ is given by

$$
\mathcal{L}_{b, K E}=\frac{1}{4} \mathcal{D}_{3}\left[\left(\nabla^{a} X^{I}\right)^{A}\left(\nabla_{a} X_{I}^{\dagger}\right)_{A}\right]_{\mid},
$$

and $\mathcal{L}_{b, V}$ is given by

$$
\mathcal{L}_{b, V}=\frac{2 \pi}{k} \mathcal{D}_{3}\left[\epsilon_{I J K L} f^{A B C D} X_{A}^{I} X_{B}^{K \dagger} X_{C}^{J} X_{D}^{L \dagger}\right] \mid .
$$

The resultant theory is given by the sum of these boundary terms with the BLG theory,

$$
\begin{aligned}
\mathcal{L}_{s, B L G}= & \mathcal{L}_{B L G}+\mathcal{L}_{b, B L G} \\
= & \frac{k}{4 \pi}\left(-\nabla^{2}+\mathcal{D}_{3}\right)\left[\frac { k } { 4 \pi } \left(f^{A B C D} \Gamma_{A B}^{a} \Omega_{a C D}+\left(\frac{1}{4} \nabla^{a} X^{I}\right)^{A B}\left(\nabla_{a} X_{I}^{\dagger}\right)_{A B}\right.\right. \\
& \left.+\frac{2 \pi}{k} \epsilon_{I J K L} f^{A B C D} X_{A}^{I} X_{B}^{K \dagger} X_{C}^{J} X_{D}^{L \dagger}\right]_{\mid} .
\end{aligned}
$$


It may be noted that only half of the supersymmetry of the original theory is preserved on the boundary. In this paper we will keep the supersymmetry generated by to $Q_{-}$and break the supersymmetry corresponding to $Q_{+}$on the boundary,

$$
\begin{aligned}
\delta_{S}^{-} \mathcal{L}_{s, B L G} & =\epsilon^{a-} Q_{-a} \mathcal{L}_{s, B L G} \\
& =\epsilon^{a-} Q_{-a} \mathcal{L}_{B L G}+\epsilon^{a-} Q_{-a} \mathcal{L}_{b, B L G} \\
& =\left[\Phi_{-}^{\prime}\left(\gamma^{3}, \Gamma^{\prime}, X^{I^{\prime}}, X^{I \dagger^{\prime}}\right)\right]_{\mid}-\left[\Phi_{-}^{\prime}\left(\gamma^{3}, \Gamma^{\prime}, X^{I^{\prime}}, X^{I \dagger^{\prime}}\right)\right]_{\mid} \\
& =0 .
\end{aligned}
$$

Thus, the supersymmetric variation of this boundary term exactly cancels the supersymmetric variation of the bulk Lagrangian, so the sum of the bulk Lagrangian and this boundary term preserves half of the supersymmetry of the original theory. It was possible to preserve the supersymmetry corresponding to $Q_{+}$in presence of the boundary if the supersymmetry corresponding to $Q_{-}$ was broken. Thus, we are able to preserve either $\mathcal{N}=(1,0)$ supersymmetry or $\mathcal{N}=(0,1)$ supersymmetry on the boundary, both not both of them.

\section{Gauge Invariance in Presence of a Boundary}

In the previous section we have seen that the original BLG theory is not supersymmetric in presence of a boundary. We have also modified the original BLG theory by adding a boundary term such that the resultant theory preserved half of the supersymmetry even in presence of the boundary. In this section we will first observe that the boundary theory is not gauge invariant. We will then modify this theory, by adding new boundary degrees of freedom, to make it gauge invariant even in presence of a boundary.

To analyse the boundary effects on the gauge invariance of the BLG theory, we first observe that the matter part of the BLG theory is gauge invariant even in presence of a boundary,

$$
\begin{aligned}
\delta \mathcal{L}_{s, K E}+\delta \mathcal{L}_{s, V} & =\left(\delta \mathcal{L}_{K E}+\delta \mathcal{L}_{b, K E}\right)+\left(\delta \mathcal{L}_{V}+\delta \mathcal{L}_{b, V}\right) \\
& =0 .
\end{aligned}
$$

However, the Chern-Simons part of the BLG theory is not gauge invariant in presence of a boundary term. This is because the infinitesimal gauge transformation of the Chern-Simons term is given by

$$
\begin{aligned}
\delta \mathcal{L}_{s, C S} & =\delta \mathcal{L}_{C S}+\delta \mathcal{L}_{b, C S} \\
& =\frac{k}{4 \pi}\left(\mathcal{D}_{3}-\nabla^{2}\right)\left[f^{A B C D}\left(D^{a} \Lambda_{A B} \omega_{a}^{A B}+C_{A B}^{C D, E F} \Gamma_{C D}^{a} \Lambda_{E F}\right) \omega_{a C D}\right]_{\mid} \\
& =-\frac{k}{4 \pi} \nabla^{2}\left[f^{A B C D}\left(\nabla^{a} \Lambda\right)_{A B} \omega_{a C D}\right]_{\mid} .
\end{aligned}
$$

Now this can be written as

$$
\delta \mathcal{L}_{s, C S}=\frac{k}{4 \pi}\left(\mathcal{D}_{3} \nabla^{a}-\left(\gamma^{\mu} \mathcal{D}_{\mu} \nabla\right)^{a}\right)\left[f^{A B C D} \Lambda_{A B} \omega_{a C D}\right] .
$$

As there is a boundary in the $x^{3}$ direction, we get

$$
\delta \mathcal{L}_{s, C S}=\frac{k}{4 \pi}\left(\mathcal{D}_{3} \nabla^{a}-\left(\gamma^{\mu} \mathcal{D}_{\mu} \nabla\right)^{a}\right)\left[f^{A B C D} \Lambda_{A B} \omega_{a C D}\right]_{\mid}
$$




$$
\sim \frac{k}{4 \pi}\left(\mathcal{D}_{3} \nabla^{a}-\left(\gamma^{3} \mathcal{D}_{3} \nabla\right)^{a}\right)\left[f^{A B C D} \Lambda_{A B} \omega_{a C D}\right]_{\mid},
$$

where $\sim$ indicates that we have neglected the total derivative contribution along directions other than $x^{3}$, as they will not contribute. Thus, the gauge transformation of this Chern-Sioms term will be,

$$
\begin{aligned}
\delta \mathcal{L}_{s, S} & =\frac{k}{4 \pi}\left(\delta_{b}^{a}-\left(\gamma^{3}\right)_{b}^{a}\right) \nabla^{\prime b}\left[f^{A B C D} \Lambda_{A B}^{\prime} \omega_{a C D}^{\prime}\right]_{\mid} \\
& =\frac{k}{2 \pi}\left(P_{-} \nabla^{\prime}\right)^{a}\left[f^{A B C D} \Lambda_{A B}^{\prime} \omega_{a C D}^{\prime}\right]_{\mid} .
\end{aligned}
$$

So, the Chern-Simons part generates a boundary term in presence of a boundary and the BLG theory is not gauge invariant in presence of a boundary.

It can however be made gauge invariant by adding new degrees of freedom on the boundary. Thus, we define a new boundary field $v_{A B}^{\prime}$ and let $v_{A B}$ its extension into the bulk. We also let $v=v_{A B} T^{A} T^{B}$. We define generate a gauge transformations for $v$ as,

$$
v \rightarrow v u^{-1} \text {. }
$$

We also define $\Gamma^{v}$ as the gauge transformation of $\Gamma$ generated by $v$

$$
\Gamma_{a}^{v}=i v \nabla_{a} v^{-1} .
$$

The potential term for the boundary field theory can now be written as

$$
\mathcal{L}_{p}=\mathcal{L}_{s v, C S}-\mathcal{L}_{s, C S}
$$

Here $\mathcal{L}_{s v, C S}$ is the Lagrangian obtained by replacing $\Gamma_{a}$ by $\Gamma_{a}^{v}$. For $v$ close to the identity, this is a actual boundary term. However, ever for large gauge transformations this can be considered as a boundary term in the sense that in the absence of a boundary, this term will have no measurable effects. The Lagrangian given by the sum of this term with the ordinal supersymmetric Chern-Simons term $\mathcal{L}_{s, C S}+\mathcal{L}_{p}$ will now be gauge invariant even in presence of a boundary,

$$
\delta \mathcal{L}_{s, C S}+\delta \mathcal{L}_{p}=0 .
$$

In fact, this potential term reduces to a generalization of the $\mathcal{N}=(1,0)$ WessZumino-Witten model [29, 30, if there is no coupling of the boundary theory to the bulk fields. Thus, this theory will be given by

$$
\begin{aligned}
\mathcal{L}_{p}= & -\frac{k}{2 \pi}\left(P_{-} \nabla^{\prime}\right)^{a} C_{A B}^{C D, E F}\left[\left[\left(v^{-1} \mathcal{D}_{+} v\right)^{A B},\left(v^{-1} \mathcal{D}_{3} v\right)_{C D}\right]\right. \\
& \left.\times\left(v^{-1} \nabla_{-a} v\right)_{E F}\right] .
\end{aligned}
$$

We can now add the following supersymmetric gauge invariant kinetic term,

$$
\mathcal{L}_{k}=-\frac{k}{2 \pi}\left(P_{-} \nabla^{\prime}\right)^{a}\left[f_{A B C D}\left(v^{\prime-1} \nabla_{-a}^{\prime} v^{\prime}\right)^{A B}\left(v^{\prime-1} \mathcal{D}_{+} v^{\prime}\right)^{C D}\right]_{\mid} .
$$

This term is gauge invariant by itself, $\delta \mathcal{L}_{k}=0$. This is a generalization of the kinetic term for the $\mathcal{N}=(1,0)$ Wess-Zumino-Witten model [29, 30]. The Lagrangian for the boundary theory will be a generalization of the $\mathcal{N}=(1,0)$ Wess-Zumino-Witten model

$$
\mathcal{L}_{b t}=\mathcal{L}_{k}+\mathcal{L}_{p}
$$


Thus, this Lagrangian also preserves the supersymmetry corresponding to $Q_{-}$, $\delta_{S}^{-} \mathcal{L}_{b t}=0$. The Lagrangian for the final gauge and supersymmetric invariant BLG theory is

$$
\mathcal{L}_{s g, B L G}=\mathcal{L}_{s, B L G}+\mathcal{L}_{b t} .
$$

This Lagrangian is invariant under gauge transformations,

$$
\delta \mathcal{L}_{s g, B L G}=0 .
$$

and preserves half of the total supersymmetry,

$$
\begin{aligned}
\delta_{S}^{-} \mathcal{L}_{s g, B L G} & =\epsilon^{a-} Q_{-a} \mathcal{L}_{s g, B L G} \\
& =0 .
\end{aligned}
$$

Thus, the BLG theory is made both gauge and supersymmetric invariant in presence of a boundary by suitable coupling it to a boundary theory. In doing so we also obtained a generalization of the gauged Wess-Zumino-Witten model, where the gauge symmetry is generated by a Lie 3-algebra rather than an ordinary Lie algebra.

\section{Conclusion}

In this paper we analysed the BLG theory in presence of boundaries in $\mathcal{N}=1$ superspace formalism. It was found that this theory is neither invariant under the supersymmetric transformations nor invariant under the gauge transformations. However by coupling this theory to a boundary theory it was made both gauge and supersymmetric invariant. The supersymmetric and gauge variations of this boundary theory exactly canceled the boundary term generated by the supersymmetric and gauge variations of this bulk theory.

In analysing this model we developed a generalization of the gauged WessZumino-Witten model, where the gauge symmetries are generated by the Lie 3 -algebra rather than an ordinary Lie algebra. It will be interesting to analyse certain features of this generalized gauged Wess-Zumino-Witten model in more detail. It is possible that this model also obeys a Kac-Moody current algebra. It will be interesting to analyse this algebra and study its properties. Furthermore, this work can be generalise to superspaces with higher amount of supersymmetry and then applied to the study of M2-branes with boundaries. As the BLG theory has also been analysed in $\mathcal{N}=2$ superspace [8, 9, and $\mathcal{N}=8$ superspace [10, 11]; it would be interesting to also analyse the the boundary BLG theory in these superspaces.

It may be remarked that before we quantise any gauge theory we have to fix a gauge. This is done at quantum level by adding a gauge fixing term and a ghost term to the original classical action. This new action is invariant under a symmetry called the BRST symmetry. This invariance of the theory under BRST symmetry is crucial to show its unitarity. The BRST symmetry of Chern-Simons theory has been thoroughly investigated [31, 32] and the BRST symmetry of $\mathcal{N}=1$ super-Chern-Simons theory has also been studied in the superspace formalism [33, 34]. The BRST invariance of ABJM theory has also been studied [35. It will be interesting to analyse the BRST symmetry for the BLG theory in presence of a boundaries in $\mathcal{N}=1$ superspace formalism. It is 
expected that the original BLG theory will not be invariant under the BRST transformations. However, a sum of the original BLG theory with the suitable boundary theory can be made BRST invariant.

\section{References}

[1] J. Bagger and N. Lambert, Phys. Rev. D 75, 045020 (2007)

[2] J. Bagger and N. Lambert, Phys. Rev. D 77, 065008 (2008)

[3] J. Bagger and N. Lambert, JHEP. 0802, 105 (2008)

[4] A. Gustavsson, Nucl. Phys. B811, 66 (2009)

[5] M. Cederwal, JHEP. 0810, 070 (2008)

[6] A. Mauri and A. C. Petkou, Phys. Lett. B 666, 527 (2008)

[7] S. V. Ketov and S. Kobayashi, Phys. Rev. D 83, 045003 (2011)

[8] M. Benna, I. Klebanov, T. Klose and M. Smedback, JHEP. 0809, 072 (2008)

[9] S. Cherkis and C. Saemann, Phys. Rev. D78, 066019 (2008)

[10] H. Samtleben and R. Wimmer, JHEP. 1002, 070 (2010)

[11] I. A. Bandos, Phys. Lett. B669, 193 (2008)

[12] P. S. Howe, N. D. Lambert and P. C. West, Nucl. Phys. B 515, 203 (1998)

[13] A. Basu and J. A. Harvey, Nucl. Phys. B 713, 136 (2005)

[14] D. S. Berman and N. B. Copland, Nucl. Phys. B 723, 117 (2005)

[15] D. S. Berman and N. B. Copland, Phys. Lett. B 639, 553 (2006)

[16] C. S. Chu and E. Sezgin, JHEP. 9712, 001 (1997)

[17] K. Ezawa, Y. Matsuo and K. Murakami, Phys. Rev. D 57, 5118 (1998)

[18] D. S. Berman, M. J. Perry, E. Sezgin and D. C. Thompson, JHEP 1004, $025(2010)$

[19] C.-S. Chu and G. S. Sehmbi, J. Phys. A 44, 134504 (2011)

[20] C.-S. Chu and D. J. Smith, JHEP. 0904, 097 (2009)

[21] O. Aharony, O. Bergman, D. L. Jafferis and J. Maldacena, JHEP. 0810, $091(2008)$

[22] O-Kab Kwon, P. Oh and J. Sohn, JHEP. 0908, 093 (2009)

[23] Bagger and N. Lambert, Phys. Rev. D 79, 025002 (2009)

[24] C.-S. Chu and D. J. Smith, JHEP. 1001, 001 (2010)

[25] D. V. Belyaev and P. van Nieuwenhuizen, JHEP. 0804, 008 (2008) 
[26] D. S. Berman and D. C. Thompson, Nucl. Phys. B 820, 503 (2009)

[27] A. Gustavsson, Nucl. Phys. B807, 315 (2009)

[28] S. J. Gates, M. T. Grisaru, M. Rocek and W. Siegel, Front. Phys. 58, 1 (1983)

[29] C. M. Hull and E. Witten, Phys. Lett. B 160, 398 (1985)

[30] C. M. Hull and B. Spence, Nucl. Phys. B 345, 493 (1990)

[31] J. Fjelstad and S. Hwang, Phys. Lett. B 466, 227 (1999)

[32] M. Chaichian and W. F. Chen, Z.Y. Zhu, Phys. Lett. B 387, 785 (1996)

[33] L. P. Colatto, M. A. De Andrade, O. M. Del Cima, D. H. T. Franco, J. A. Helayel-Neto and O. Piguet, J. Phys. G 24, 1301 (1998)

[34] C. P. Constantinidis, O. Piguet and W. Spalenza, Eur. Phys. J. C 33, 443 (2004)

[35] M. Faizal, Phys. Rev. D 84, 106011 (2011) 